\title{
Influence of the GEO satellite orbit error fluctuation correction on the BDS WADS zone correction
}

\author{
Binghao Wang ${ }^{1}$, Jianhua Zhou'* ${ }^{\text {* }}$ Bin Wang ${ }^{2}$, Dianwei Cong ${ }^{3}$ and Hui Zhang ${ }^{4}$
}

\begin{abstract}
Decimeter-level service is provided by the BeiDou satellite navigation system wide area differential service (BDS WADS) for users who collect carrier phase measurements. However, the fluctuations in Geostationary Earth Orbit (GEO) satellite orbit errors reduce the spatial correlation of orbit errors. These fluctuations not only decrease the accuracy and stability of zone correction service provided by BDS WADS, but also shorten its effective range. In this paper, we proposed an algorithm to weaken the influence of GEO satellite orbit error fluctuations and verified the method using data from eight sparsely distributed zones. The results show that orbit errors can be stabilized using orbit fluctuation corrections, and the positioning precision and stability of the BDS WADS can be improved simultaneously. Under normal circumstances, the horizontal and vertical positioning accuracy of users within $1000 \mathrm{~km}$ from the center of the zone can reach $0.19 \mathrm{~m}$ and $0.34 \mathrm{~m}$. Furthermore, the effective range is increased. The positioning performance within $1800 \mathrm{~km}$ could reach $0.24 \mathrm{~m}$ and $0.38 \mathrm{~m}$ for the horizontal and vertical components, respectively.
\end{abstract}

Keywords: Wide area differential service, Decimeter-level service, Zone correction, GEO orbit error, Effective range

\section{Introduction}

The BDS space segment is a hybrid constellation of GEO, Inclined Geosynchronous Satellite Orbit (IGSO) and Medium Earth Orbit (MEO) satellites. The core constellation of the BDS-3 consists of 24 MEO satellites (Yang et al. 2019a, b) and was completed after four satellites joined the system on December 17, 2019. Soon after, the 54th BDS satellite was launched into orbit on March 9, 2020. And the final GEO will be launched in June, 2020. In addition, all the services designed (Yang et al. 2019a, b) for the BDS-3 (fundamental service, the satellite-based augmentation service, precise point positioning service, short message communication service and search and rescue service) will be available in 2020 .

As the BDS space segment has continuously improved, its superior service performance has been gradually

\footnotetext{
*Correspondence: zjh_sn@126.com

1 Beijing Navigation Center, Beijing 100094, China

Full list of author information is available at the end of the article
}

highlighted and had drawn worldwide attention (Wang et al. 2019; Yang et al. 2018, 2020; Zhang et al. 2019a, b). The latest research results show that the precision of the BDS satellite's orbit has been tremendously improved with the addition of an inter-satellite link (Yang et al. 2019a, b, 2020). The radial accuracy of the broadcast orbit can reach $10 \mathrm{~cm}$, as evaluated by the satellite laser ranging (SLR) method, and the signal-in-space user range error (SISURE) is better than $0.5 \mathrm{~m}$ (Yang et al. 2020). But precise orbit determination of GEO satellites remains to be a challenge.

In addition to the upgrade of the fundamental service, the augmentation service, which uses the differential method, has effectively improved the performance of GNSS positioning, navigation, and has also produced great social benefits ( $\mathrm{Li}$ et al. 2020). The evolution of the differential service can be divided into three phases: (1) the pseudo range wide area differential service, which is based on widely and sparsely distributed ground stations, such as the Wide Area Augmentation System (WAAS) of the United States (Yang et al. 
2017), the European Geostationary Navigation Overlay Service (EGNOS) of Europe (Ventura-Traveset et al. 2015), and the System for Differential Corrections and Monitoring (SDCM) of Russia (Lu et al. 2014), is widely used in precision approaches of civil aviation because of its high integrity (Yu et al. 2019). The BDS Satellite Based Augmentation Service (BDSBAS) is currently undergoing testing and may open soon ( $\mathrm{Li}$ et al. 2020). (2) The high precision local area differential service, in which densely distributed monitoring stations and high capacity communications are required for model construction and parameters broadcast. Network Real Time Kinematic (NRTK) systems can provide centimeter-level positioning precision in seconds and have greatly promoted industrialization. (3) Satellite-Station differential services, such as Trimble RTX (Krzyżek 2014), StarFire (Dai et al. 2016), Atlas, etc., make use of globally distributed ground monitoring networks to realize the effective separation and modeling of error sources. Using the correction information broadcasted by communication satellites, satellite-station services can provide global real-time precise point positioning, which has broad application prospects.

To effectively improve the performance of the BDS, the operation control segment of the BDS established a wide area differential system as an alternative to the BDSBAS. The BDS Wide Area Differential Service (WADS) was released in January 2017 and declared decimeter-level positioning accuracy for users collecting dual-frequency carrier phase observations. Differential corrections provided by BDS WADS consist of Equivalent Satellite Clock (ESC) corrections, ionosphere grid corrections, orbit corrections and zone corrections. These corrections were generated based on the BDS-2 constellation and monitoring network (Chen et al. 2017; Yang 2017; Zhang 2017). All corrections are broadcasted by the BDS GEO satellites. Users can obtain decimeter-level positioning precision by receiving and properly using the aforementioned four types of corrections (Chen et al. 2015; Wang et al. 2017).

Fundamental of zone correction enhancing positioning is based on the spatial correlations of orbit error, atmosphere delay and other error sources between the user and reference stations (Zhang 2017). Zone corrections can also be called comprehensive corrections. They are broadcasted over a 36-s period for users in the service zone to eliminate errors in carrier phase measurements for quicker convergence and higher precision positioning.

Zone correction is a type of Observation Space Representation (OSR) correction, and the service performance is highly related to the distance between the user and center of zones. For the real-time high precision augmentation service, two main aspects of user positioning service promotion can be concluded as: (1) stable performance at a certain distance between the user and the center of the zone; and (2) the maximization of the effective range at a given accuracy requirement.

As a significant error source in wide area differential positioning, orbit error inevitably influences the performance of the zone correction service. Fluctuations greater than $10 \mathrm{~m}$ were observed in the GEO broadcast orbit during several periods, which may have introduced disadvantages into zone correction service. An algorithm aiming at eliminating orbit error fluctuations was proposed in this contribution. In addition, the algorithm was verified using real measurements. After correction, the influence of the GEO orbit error fluctuations on the zone correction is considerably alleviated. The linear correlation between the positioning performance attenuation and the user-reference distance is more explicit, and the effective range of the zone correction is widely increased.

\section{Influence on the zone correction}

The positioning model based on the BDS WADS zone correction was elaborately demonstrated in Chen et al. (2018) and Zhang et al. (2017), and ionosphericfree combinations (B1/B2, B1/B3 or B2/B3) are recommended. By augmentation of the zone correction, ionospheric-free phase observations on the user side can be expressed as follows (Chen et al. 2018):

$$
\begin{aligned}
L_{u}^{\prime s}= & \rho_{u}^{\prime s}+\left(d \rho_{u}^{s}-d \rho_{r}^{s}\right)+c \cdot\left(d t_{u}-d t_{r}\right) \\
& +\left(N_{u}^{s}-N_{r}^{s}\right)+d T_{u}-d T_{r}+\varepsilon_{L}
\end{aligned}
$$

where $\rho_{u}^{\prime s}$ is the corrected distance between the satellite $s$ and station $u$ after application of the zone correction. In addition, $c$ is the speed of light in vacuum. The satellite clock has been eliminated. The clock offset of the reference station $d t_{r}$ would be absorbed by the user clock offset $d t_{u}$, and has no influence on positioning. The ambiguity offset of the reference station $N_{r}^{s}$ would be absorbed into the ambiguity of the user $N_{u}^{s}$ and would not place an extra burden on the parameter estimation process if no cycle slip occurs in the reference station carrier-phase observations. $d T_{u}-d T_{r}$ is the difference in the troposphere delay between the user and reference station, and it can be regarded as a constant over a short period and can also be absorbed in the float ambiguities on the user's side. $d \rho_{u}^{s}$ and $d \rho_{r}^{s}$ are the projection of the orbit errors of the satellite $s$ along the line of sight (LOS) for the reference station and the user, respectively, and consist of radial, along and cross components. The ESC correction performed before the application of zone correction corrects the radial component of the orbit error together with the satellite clock bias. Therefore, the residual orbit 
error that exists after the ESC correction is mainly composed of the along and cross components, as follows:

$$
d \rho_{r}^{s}=\cos \left(\alpha_{r}^{s}\right) \cdot a^{s}+\cos \left(\beta_{r}^{s}\right) \cdot c^{s}
$$

where $\alpha$ and $\beta$ are the angles between the along and cross directions and the LOS, respectively. Subscript $r$ is the reference station mark, while superscript $s$ represents the satellite. $a^{s}$ and $c^{s}$ represent the residual orbit errors of the satellite $s$ in the along and cross components, respectively. $d e l_{\text {orb }}=d \rho_{r}^{s}-d \rho_{u}^{s}$ is set to be the difference in the orbit error projection between the user and the reference station. Similar to the troposphere delay, del $l_{\text {orb }}$ could be absorbed in the ambiguity as a constant value and introduces no disadvantage to parameter estimation when it is a stable value. However, the positioning precision and stability will deteriorate if $d_{e l} l_{o r b}$ is not stable.

If Eq. (2) is substituted into $d_{e l} l_{o r b}$, the resulting equation is as follows:

$$
\begin{aligned}
d e l_{o r b}= & d \rho_{r}^{s}-d \rho_{u}^{s}=a^{s} \cdot\left(\cos \left(\alpha_{r}^{s}\right)-\cos \left(\alpha_{u}^{s}\right)\right) \\
& +c^{s} \cdot\left(\cos \left(\beta_{r}^{s}\right)-\cos \left(\beta_{u}^{s}\right)\right)
\end{aligned}
$$

With 18 zones and an effective range set to $1000 \mathrm{~km}$, the WADS zone correction service can realize $100 \%$ coverage of China (Zhang 2017). At a distance of $1072 \mathrm{~km}$, two stations in Beijing and Wuhan are selected to demonstrate the influence of the angles $\alpha$ and $\beta$ on $\alpha_{e} l_{o r b}$. Using the results of February 20, 2018 as an example, variations in $\cos \left(\alpha_{r}^{s}\right)-\cos \left(\alpha_{u}^{s}\right)$ and $\cos \left(\beta_{r}^{s}\right)-\cos \left(\beta_{u}^{s}\right)$ are shown in Fig. 1.

Figure 1 shows that the coefficient composed of angles is limited to \pm 0.023 , and the difference in $10 \mathrm{~min}$ is constrained to $4 \times 10^{-5}$. Therefore, the instability of $\operatorname{del}_{\text {orb }}$ is mainly caused by fluctuations in $a^{s}, c^{s}$.

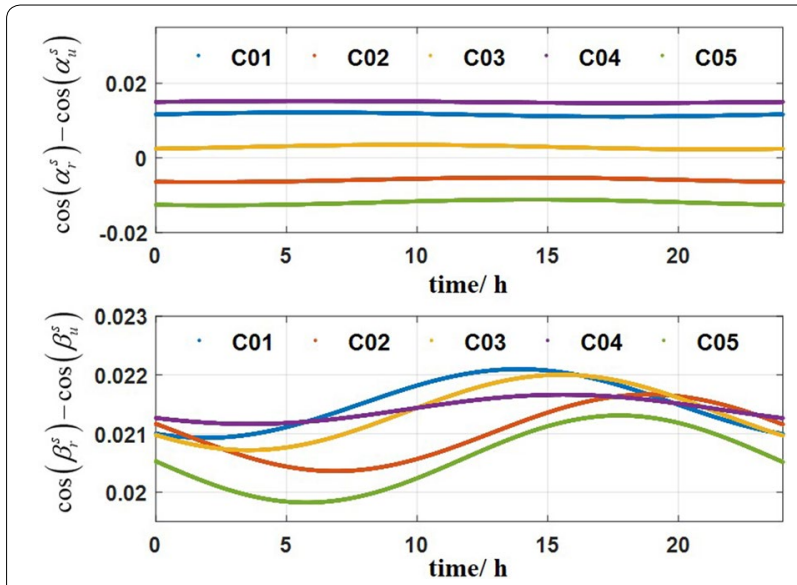

Fig. 1 Variations in $\cos \left(\alpha_{r}^{s}\right)-\cos \left(\alpha_{u}^{s}\right)$ (top) and $\cos \left(\beta_{r}^{s}\right)-\cos \left(\beta_{u}^{s}\right)$ (bottom) calculated using data from Beijing and Wuhan from February 20, 2018
Massive experiments were conducted to establish the correlation between the position accuracy and orbit error stability. If numerous GEO satellites are assumed to be involved in data processing, the standard deviation (STD) values of each GEO satellite are computed as follows:

$$
\sigma^{j}=\sqrt{\frac{1}{N} \sum_{i=1}^{N}\left(\operatorname{del}_{o r b, j}{ }^{i}-\operatorname{mean}\left(\operatorname{del}_{o r b, j}\right)\right)}
$$

where $N$ is the length of $d e l_{o r b, j}$ series and mean $(*)$ is the averaging operation. The maximum value of $\sigma^{j}(j=1 \ldots m)$ is then established to be the index of the orbit error stability description. The variation of the accuracy of the B1/B2 dual-frequency positioning relative to the stability of $d_{e l} l_{\text {orb }}$ is shown in Fig. 2.

Obviously, the instability of $d_{e} l_{o r b}$ caused by the orbit error in the along and cross directions will decrease the positioning performance of the B1/B2 dual-frequency positioning enhanced by the zone correction.

\section{Demonstration of the GEO orbit error fluctuations and the correction algorithm}

Due to the regional distribution of the BDS monitoring network, it has been difficult to precisely determine the orbit of the GEO satellites, especially in the along and cross components. Using the final products of GFZ as a reference, the broadcast GEO orbit error in the first half of 2018 was calculated and large fluctuations were found. Large fluctuations in the cross and along components are shown in Fig. 3.

In Fig. 3, 006 C02 stands for C02 in DOY (day of year) 6. Fluctuations greater than $20 \mathrm{~m}$ are shown in the right panel of Fig. 3. In the positioning procedure, the worst

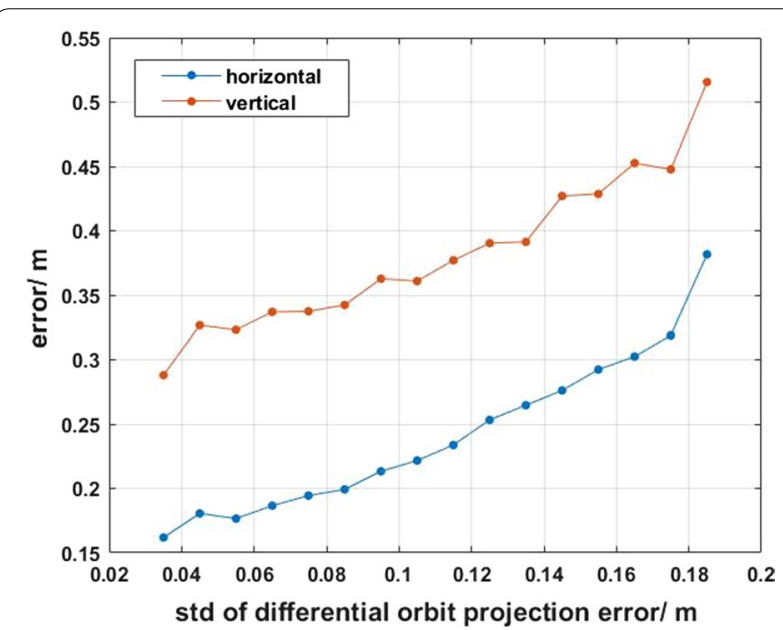

Fig. 2 Model of the correlation between the dual-frequency precise point positioning accuracy (RMS) enhanced by the zone correction and orbit error stability 

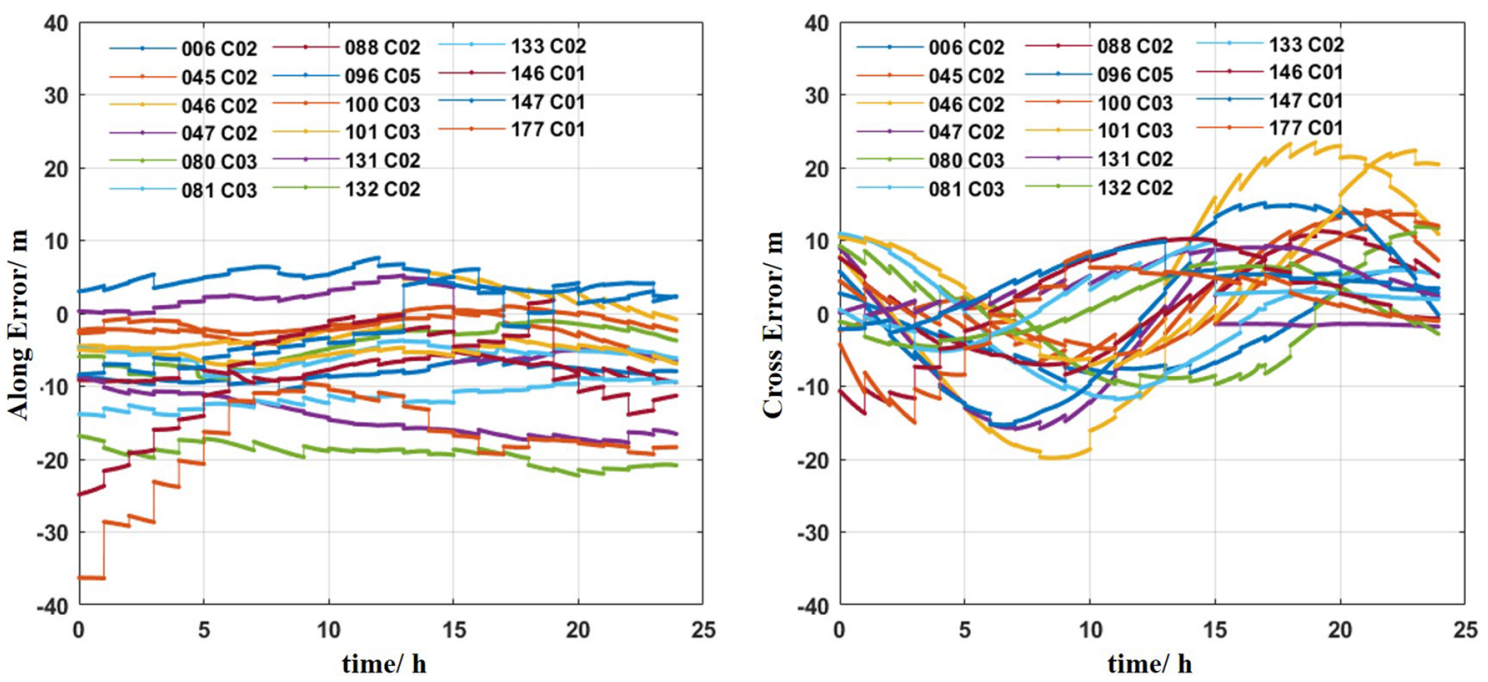

Fig. 3 Fluctuations in the along (left) and cross (right) components founded in the BDS GEO broadcast ephemeris during the first half of 2018

measurement will be the bottleneck in the high precision differential positioning. Therefore, as shown in Fig. 3, the fluctuations in the broadcast GEO orbit will limit the promotion of the differential positioning precision.

Fluctuations in the GEO broadcast orbit would decrease the accuracy and stability of the BDS positioning and make the effective range of the zone correction more ambiguous. To ensure a high performance of the zone correction, fluctuations in the GEO broadcast orbit error should be reasonably corrected.

An algorithm for the GEO orbit fluctuation correction was proposed. In this algorithm, the orbit error variations in the cross and along directions between two epochs were estimated based on zone corrections. The results were then used to compensate the GEO orbit error.

The orbit error, clock bias, troposphere modeling residual and ambiguity offset are the main components of the zone correction (Zhang 2017). The difference method is used to eliminate the clock bias and ambiguity offset and to weaken the influences of the troposphere modeling residual, then orbit component in zone corrections can be extracted. Furthermore, the variations in the orbit error can be estimated using differenced zone corrections and the least square method.

To conduct the difference operation, one GEO satellite is selected as the reference satellite and the double-differenced zone corrections are formed among multiple zones and satellites. After the double-differenced operation, the clock bias is eliminated, and the errors that remain in the double-differenced zone corrections are the double-differenced orbit error, troposphere residual and ambiguity offset, as follows:

$$
\begin{aligned}
\nabla \Delta \delta L_{r, u}^{i, j} & =\delta L_{u}^{j}-\delta L_{r}^{j}-\delta L_{u}^{i}+\delta L_{r}^{i} \\
& =\nabla \Delta o r b_{r, u}^{i, j}+\nabla \Delta \delta T_{r, u}^{i, j}+\lambda \cdot \nabla \Delta N_{r, u, 0}^{i, j} .
\end{aligned}
$$

The double-differenced orbit error can be expanded as follows:

$$
\begin{aligned}
\nabla \Delta o r b_{r, u}^{i, j}= & o r b_{u}^{j}-o r b_{u}^{i}-o r b_{r}^{j}+o r b_{r}^{i} \\
= & a^{j} \cdot\left(\cos \left(\alpha_{u}^{j}\right)-\cos \left(\alpha_{r}^{j}\right)\right) \\
& +c^{j} \cdot\left(\cos \left(\beta_{u}^{j}\right)-\cos \left(\beta_{r}^{j}\right)\right) \\
& -a^{i} \cdot\left(\cos \left(\alpha_{u}^{i}\right)-\cos \left(\alpha_{r}^{i}\right)\right) \\
& -c^{i} \cdot\left(\cos \left(\beta_{u}^{i}\right)-\cos \left(\beta_{r}^{i}\right)\right) .
\end{aligned}
$$

Furthermore, differences between the adjacent epochs (interval: $600 \mathrm{~s}$ ) are calculated to eliminate the ambiguity offset and troposphere residual. Using the analysis shown in Fig. 1, the differences in the values of $\cos \left(\alpha_{r}^{s}\right)-\cos \left(\alpha_{u}^{s}\right)$ and $\left(\cos \left(\beta_{r}^{s}\right)-\cos \left(\beta_{u}^{s}\right)\right)$ between the epochs could be ignored. The triple-differenced zone correction can be expressed as follows:

$$
\begin{aligned}
\Delta \nabla \Delta \delta L_{r, u}^{i, j}(t, t-1)= & \nabla \Delta \delta L_{r, u}^{i, j}(t)-\nabla \Delta \delta L_{r, u}^{i, j}(t-1) \\
= & a_{t, t-1}^{i} \cdot\left(\cos \left(\alpha_{u}^{i}(t)\right)-\cos \left(\alpha_{r}^{i}(t)\right)\right) \\
& +c_{t, t-1}^{i} \cdot\left(\cos \left(\beta_{u}^{i}(t)\right)-\cos \left(\beta_{r}^{i}(t)\right)\right) \\
& -a_{t, t-1}^{j} \cdot\left(\cos \left(\alpha_{u}^{j}(t)\right)-\cos \left(\alpha_{r}^{j}(t)\right)\right) \\
& -c_{t, t-1}^{j} \cdot\left(\cos \left(\beta_{u}^{j}(t)\right)-\cos \left(\beta_{r}^{j}(t)\right)\right)
\end{aligned}
$$


where $a_{t, t-1}^{i}$ and $a_{t, t-1}^{j}, c_{t, t-1}^{i}$ and $c_{t, t-1}^{j}$ stand for orbit error variations of satellite $i$ and $j$ in the along and cross directions between epochs $t$ and $t-1$. With multiple triple-differenced zone corrections, orbit error variations can be estimated as follows:

$$
\left[\begin{array}{c}
\text { resi }_{1} \\
\vdots \\
\text { resi }_{n}
\end{array}\right]_{n \times 1}=\left[\begin{array}{ccc}
h_{1,1} & \ldots & h_{1,10} \\
\vdots & \ddots & \vdots \\
h_{n, 1} & \ldots & h_{n, 10}
\end{array}\right]_{n \times 10} \cdot\left[\begin{array}{c}
x_{1} \\
\vdots \\
x_{10}
\end{array}\right]_{10 \times 1}
$$

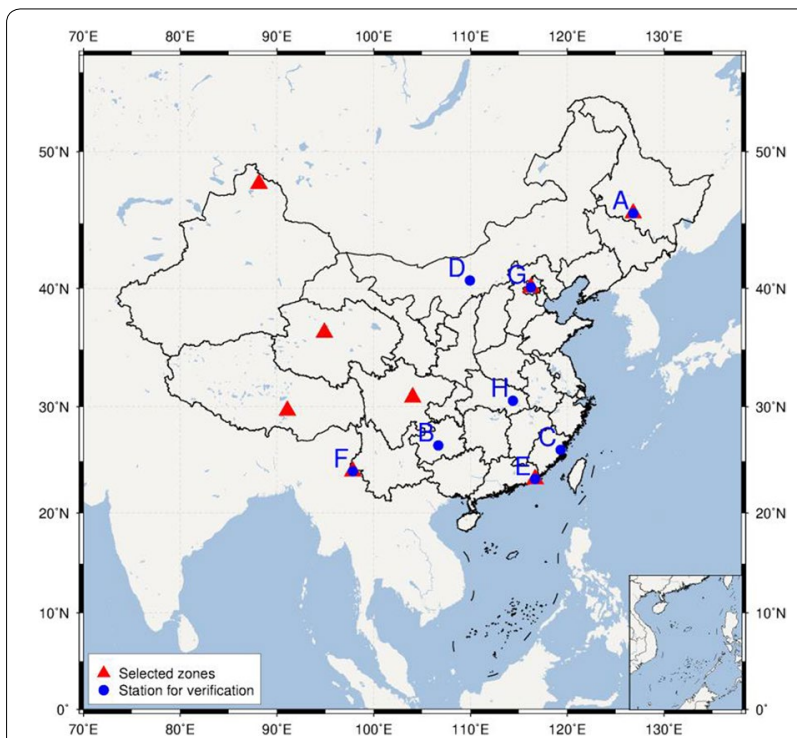

Fig. 4 Distribution of the 8 selected zones (red triangle) and 8 positioning stations (blue point) used for verification where resi $_{k}$ represents the $k$ th triple-differenced zone correction residual whose reference and slave zones are $r$ and $u$, and the reference and slave satellites are $i$ and $j$. On the right side of Eq. (8), $x_{2 l-1}$ and $x_{2 l}$ are the variations in the along and cross components of satellite $l$, respectively, where $l=1 \ldots 5$. In each row of the design matrix, coefficients in $h_{k, 1} \ldots h_{k, 10}$ remain zero, except the 4 coefficients listed below:

$$
\begin{aligned}
h_{k, 2 \times i-1} & =\cos \left(\alpha_{u}^{i}\right)-\cos \left(\alpha_{r}^{i}\right) \\
h_{k, 2 \times i} & =\cos \left(\beta_{u}^{i}\right)-\cos \left(\beta_{r}^{i}\right) \\
h_{k, 2 \times j-1} & =-\cos \left(\alpha_{u}^{j}\right)+\cos \left(\alpha_{r}^{j}\right), j \neq i . \\
h_{k, 2 \times j} & =-\cos \left(\beta_{u}^{j}\right)+\cos \left(\beta_{r}^{j}\right)
\end{aligned}
$$

The solutions of Eq. (8) could then be used to correct the GEO broadcast orbit error.

\section{Algorithm verification}

To verify the algorithm proposed previously, 8 sparsely distributed zones on the mainland of China are chosen to estimate the orbit error fluctuation, and the distribution of the selected zones is shown in Fig. 4.

To increase the precision and reliability of the GEO orbit error correction, $5^{\circ}$ in longitude or latitude was set as a threshold in zone selection.

The orbit error variations in the periods shown in Fig. 3 are estimated. The broadcast GEO orbit errors in the along and cross components are then corrected with the estimated results. The $\mathrm{C} 03$ orbit correction results of the DOY 80 and DOY 81 are shown as examples.

The along and cross components of the $\mathrm{C} 03$ broadcast orbit error in Fig. 5 show apparent fluctuations. After
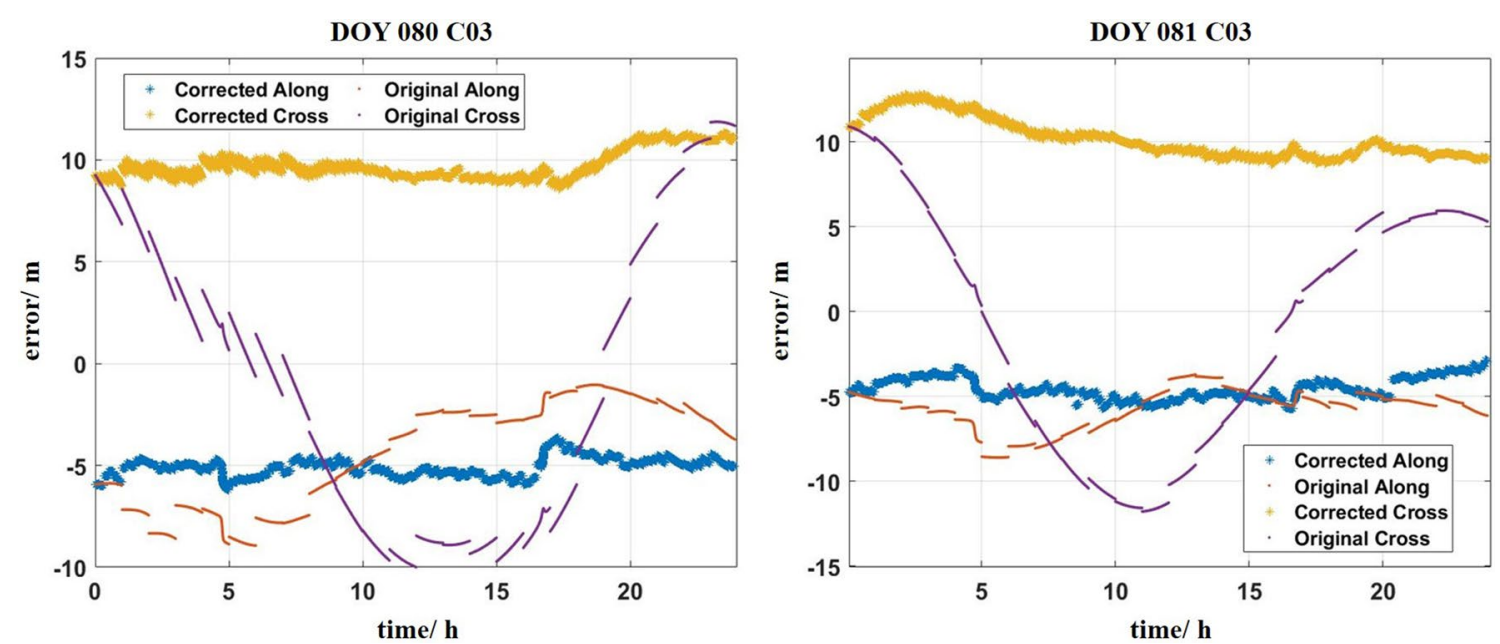

Fig. 5 Orbit fluctuation corrected results of C03 in DOY 080 (left) and DOY 081 (right) 
Table 1 Stations used for verification and corresponding zones

\begin{tabular}{llll}
\hline Zone & Station & Distance $(\mathbf{k m})$ & $\begin{array}{l}\text { Experiment } \\
\text { number }\end{array}$ \\
\hline Beijing & A & 1060 & 1 \\
& C & 1580 & 2 \\
\multirow{6}{*}{ Wuhan } & H & 1072 & 3 \\
& A & 1990 & 4 \\
& B & 880 & 5 \\
& D & 1190 & 6 \\
& E & 830 & 7 \\
& F & 1780 & 8 \\
& G & 1072 & 9 \\
\hline
\end{tabular}
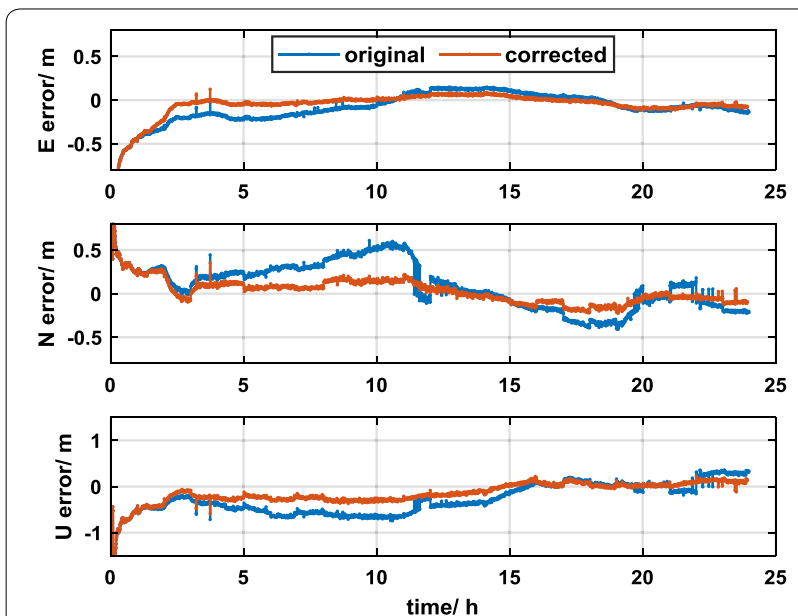

Fig. 6 Positioning results with (corrected) and without (original) the fluctuation correction

correction, the fluctuations are effectively removed. A constant offset exists in the corrected orbit error, but it will transition into constant value in differential positioning and the results will not be affected.
Observation data from 8 stations are shown in Fig. 4 and corrections from the 2 zones were selected to demonstrate the influence of the orbit correction on the zone correction augmentation positioning. The B1/B2 ionospheric-free combination was used in data processing and the weights of the GEO and IGSO/MEO observations were set to be $0.5: 1$. The distances between the positioning stations and corresponding zones are shown in Table 1.

Using the results from experiment 9 as an example, the results are shown in Fig. 6.

In Fig. 6, the original and corrected terms indicate without and with orbit correction during positioning, respectively. The results show that the precision and stability of the positioning are obviously improved when correction is applied to the GEO broadcast orbit. However, no apparent promotion is observed in the first $2 \mathrm{~h}$ when the ambiguities are not all convergent. The reason is that in the convergence period, the code measurements contribute more to the estimation of parameters than the carrier phase observation does. The orbit error correlated bias was overwhelmed by the code observation error. However, after convergence, the carrier phase observation error and orbit error correlated bias have comparable magnitudes. Therefore, the orbit error fluctuation mainly affects the precision and stability after convergence. All results are summarized in Table 2.

The results in Table 2 show that the level of influence is correlated with the distance between the station and center of the zone. For example, in experiments 2 and 4, the worst results are achieved. However, a much better result is obtained in experiment 8 , even though the distance between station $\mathrm{F}$ and the center of the zone was $1780 \mathrm{~km}$. The explanation for this discrepancy is that if the apparent fluctuation exists in the cross direction, the $c^{s} \cdot\left(\cos \left(\beta_{r}^{s}\right)-\cos \left(\beta_{u}^{s}\right)\right)$ component in Eq. (3) plays a dominant role, which reduces the effective range in the latitude direction more than in the longitude direction.

Table 2 Positioning results with and without the orbit error fluctuations correction (unit: $\mathbf{m}$ )

\begin{tabular}{|c|c|c|c|c|c|c|c|}
\hline \multirow[t]{2}{*}{ Zone } & \multirow[t]{2}{*}{ Station } & \multicolumn{3}{|c|}{ Original } & \multicolumn{3}{|c|}{ Corrected } \\
\hline & & $\mathrm{E}$ & $\mathrm{N}$ & $U$ & $\mathrm{E}$ & $\mathrm{N}$ & $U$ \\
\hline \multirow[t]{3}{*}{ Beijing } & A & 0.08 & 0.20 & 0.31 & 0.05 & 0.08 & 0.17 \\
\hline & C & 0.16 & 0.35 & 0.63 & 0.10 & 0.11 & 0.35 \\
\hline & $\mathrm{H}$ & 0.13 & 0.16 & 0.45 & 0.05 & 0.06 & 0.21 \\
\hline \multirow[t]{6}{*}{ Wuhan } & A & 0.34 & 0.46 & 0.57 & 0.13 & 0.16 & 0.34 \\
\hline & B & 0.06 & 0.09 & 0.13 & 0.03 & 0.05 & 0.09 \\
\hline & D & 0.14 & 0.20 & 0.30 & 0.08 & 0.12 & 0.15 \\
\hline & $E$ & 0.13 & 0.16 & 0.33 & 0.06 & 0.05 & 0.21 \\
\hline & F & 0.13 & 0.13 & 0.28 & 0.07 & 0.07 & 0.19 \\
\hline & G & 0.12 & 0.27 & 0.40 & 0.05 & 0.10 & 0.19 \\
\hline
\end{tabular}


Consider the scenario in which the point $30^{\circ} \mathrm{N}, 116^{\circ}$ $\mathrm{E}$ is set as the reference point and the area $20^{\circ}-40^{\circ} \mathrm{N}$, $100^{\circ}-130^{\circ} \mathrm{E}$ is set as the test area. The variations in the orbit error in the periods shown in Fig. 3 are estimated. If the test area is divided by $1^{\circ} \times 0.5^{\circ}$ and grid points are set to be virtual users, the STD values of $d_{e} l_{\text {orb }}$ between the virtual users and the reference point are calculated based on the original and corrected GEO errors. The STDs are then sorted and grouped into groups that had similar distances between the user and the reference point. Figure 7 shows the changes in the average STD of each group and the distance between the station and the center of the zone.

Without the orbit fluctuation correction, the STD values of $d_{e} l_{\text {orb }}$ relative to distance show poor stability, which will cause a complex pattern of precision attenuation as the distance increases, which is not desirable for wide area differential service.

After correction, the stability of the orbit error is improved, which will contribute to a lower correlation of the orbit projection error between the user and its reference station. Namely, users at the same distance will gain similar precision and stability. In addition, the correlation between the STD of the del $_{\text {orb }}$ and the distance presents an approximately linear relationship, which indicates that for certain accuracy demands, the effective area of the zone correction is more regular (a circle with its center at the center of the zone) than that in the original condition. At the same distance, the STDs of $d_{e l} l_{\text {orb }}$ are much more concentrated after correction, which indicates a higher precision and wider effective range.

As mentioned above, at an effective range of $1000 \mathrm{~km}$, 18 zones could realize $100 \%$ coverage of mainland of China. Based on the principle of proximity, the distance between the user and the center of the zone is limited to $1000 \mathrm{~km}$ if the service regularly operates. As shown in Fig. 7, with correction, the STDs of del orb within $1000 \mathrm{~km}$ are up to $0.08 \mathrm{~m}$. Similar to the relationship between the STD of $d_{e} l_{o r b}$ and the positioning precision shown in Fig. 2, the B1/B2 dual-frequency user can achieve $0.19 \mathrm{~m}$ and $0.34 \mathrm{~m}$ positioning precisions for the horizontal and vertical components, respectively.

If the distance expands to $1800 \mathrm{~km}$, the STD of del orb is still under $0.12 \mathrm{~m}$. Compared with those in Fig. 2, the horizontal and vertical positioning accuracies can still reach $0.24 \mathrm{~m}$ and $0.38 \mathrm{~m}$ within $1800 \mathrm{~km}$. The effective range is widely expanded.

\section{Summary and conclusion}

The release of the BDS WADS increases the precision of a user's positioning to the decimeter scale. However, fluctuations in the GEO broadcast orbit are disadvantageous for the positioning precision and stability. In this study, an algorithm to estimate fluctuations in the GEO orbit errors was proposed and verified using real measurements.

With the fluctuation correction, the orbit error of GEO was stabilized and stability of differential orbit projection error between user and station was improved effectively, and higher positioning precision can be achieved. In normal service, users in $1000 \mathrm{~km}$ range from zone center can acquire a precision of $0.19 \mathrm{~m}, 0.34 \mathrm{~m}$ for horizontal and vertical components with B1/B2 dual-frequency combination observations under the augmentation of zone correction.
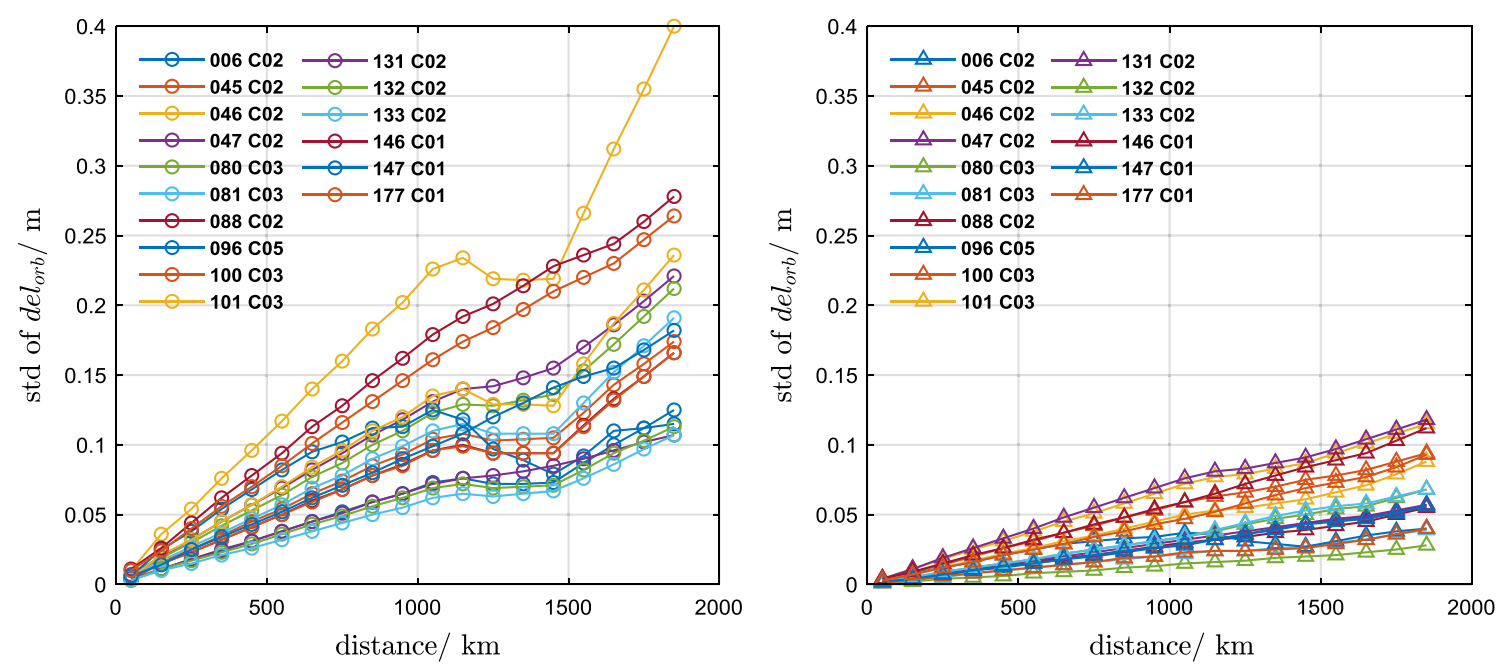

Fig. 7 STDs of del orb in the test area vary with distance from the center of the zone, before (left) and after (right) the fluctuation correction 
After the GEO broadcast orbit corrections are applied, the spatial correlation of the orbit error is effectively reduced, and the relationship between the orbit projection error and the positioning accuracy tends to be linear. The pattern of accuracy attenuation with increased distance becomes more consistent. The effective range is widely expanded and decimeter-level positioning precision is available within $1800 \mathrm{~km}$, which will guarantee desirable precision, stability and continuity for WADS users.

\section{Acknowledgements}

This study is supported by the National Natural Science Funds of China (Grant No. 41604032). We sincerely thank the GFZ, which provided the BDS precise orbit products,and the authors also thank Wang Ahao and Professor Chen Junping for their helpful comments and participation.

\section{Authors' contributions}

BW and JZ conceived and developed the algorithms; $\mathrm{HZ}$ and DC performed the experiments; BW and BW analyzed the data; and BW wrote the paper. All authors read and approved the final manuscript.

\section{Funding}

This study is supported by the National Natural Science Funds of China (Grant No. 41604032).

\section{Availability of data and materials}

The datasets used and analyzed during the current study are available from the corresponding author upon reasonable request.

\section{Competing interests}

The authors declare that they have no competing interests.

\section{Author details}

${ }^{1}$ Beijing Navigation Center, Beijing 100094, China. ${ }^{2}$ Shanghai Astronomical Observatory, Chinese Academy of Sciences, Shanghai 200030, China. ${ }^{3}$ Information Engineering University, Zhengzhou 450052, China. ${ }^{4}$ National Defense University Joint Operations College, Shijiazhuang 050001, China.

Received: 6 November 2019 Accepted: 6 May 2020

Published online: 08 June 2020

\section{References}

Chen, J., Hu, Y., Zhang, Y., \& Zhou, J. (2017). Preliminary evaluation of performance of BeiDou satellite-based augmentation system. Journal of Tongji University (Natural Science), 045(007), 1075-1082.

Chen, J., Zhang, Y., Yang, S., \& Wang, J. (2015). A new approach for satellite based GNSS augmentation system: From sub-meter to better than 0.2 meter era. In The ION 2015 Pacific PNT Meeting, Honolulu, Hawaii (pp. 180-184).

Chen, J., Zhang, Y., Zhou, J., Yang, S., Hu, Y., \& Chen, Q. (2018). Zone correction: A SBAS differential correction model for BDS decimeter-level positioning. Acta Geodaetica et Cartographica Sinica, 47(9), 1161-1170.
Dai, L., Chen, Y., Lie, A., Zeitzew, M., \& Zhang, Y. (2016). StarFire ${ }^{\text {TM }}$ SF3: Worldwide centimeter-accurate real time GNSS positioning. In The International technical meeting of the Satellite Division of the Institute of Navigation, Portland (pp. 1-6).

Krzyżek, R. (2014). Precision analysis of Trimble RTX surveying technology with xFill function in the context of obtained conversion observations. Reports on Geodesy \& Geoinformatics, 97(1), 47-70.

Li, R., Zheng, S., Wang, E., Chen, J., Feng, S., Wang, D., et al. (2020). Advances in BeiDou Navigation Satellite System (BDS) and satellite navigation augmentation technologies. Satellite Navigation, 1(1), 12. https://doi. org/10.1186/s43020-020-00010-2.

Lu, L., Ma, Y., \& Chen, H. (2014). The current status and development of SDCM. In The 5th China satellite navigation conference, Nanjing (pp. 1-6).

Ventura-Traveset, J., Echazarreta, C. L. D., Lam, J. P., \& Flament, D. (2015). An introduction to EGNOS: The European Geostationary Navigation Overlay System. Dordrecht: Springer.

Wang, H., Chen, J., \& Zhang, Y. (2017). Realization of BDS single-frequency point positioning of sub-meter accuracy. In The 8th China satellite navigation conference, Shanghai (pp. 1-6).

Wang, M., Wang, J., Dong, D., Meng, L., Chen, J., Wang, A., et al. (2019). Performance of BDS-3: Satellite visibility and dilution of precision. GPS Solutions, 23(2), 56.

Yang, S. (2017). Research on BDS decimeter level SBAS and its performance assessment (pp. 1-137). Shanghai: University of Chinese Academy of Science.

Yang, Y., Gao, W., Guo, S., Mao, Y., \& Yang, Y. (2019a). Introduction to BeiDou-3 navigation satellite system. Annual of Navigation, 66(1), 7-18.

Yang, T., Li, R., Chen, J., \& Gao, W. (2017). WAAS performance evaluation. In The 8th China satellite navigation conference, Shanghai (pp. 1-6).

Yang, Y., Mao, Y., \& Sun, B. (2020). Basic performance and future developments of BeiDou global navigation satellite system. Satellite Navigation. https:// doi.org/10.1186/s43020-019-0006-0.

Yang, Y., Xu, Y., Li, J., \& Yang, C. (2018). Progress and performance evaluation of BeiDou global navigation satellite system: Data analysis based on BDS-3 demonstration system. Science China-Earth Sciences, 61(5), 614-624.

Yang, Y., Yang, Y., Hu, X., Tang, C., Zhao, L., \& Xu, J. (2019b). Comparison and analysis of two orbit determination methods for BDS-3 satellites. Acta Geodaetica et Cartographica Sinica, 48(7), 831-839.

Yu, S., Zhang, X., Guo, F., Li, X., Pan, L., \& Ma, F. (2019). Recent advances in precision approach based on GNSS. Acta Aeronautica et Astronautica Sinica, 40(3), 22200-022200.

Zhang, Y. (2017). Research on real-time high precision BeiDou positioning service system (pp. 1-181). Shanghai: Tongji University.

Zhang, Y., Chen, J., Yang, S., \& Chen, Q. (2017). Initial Assessment of BDS Zone Correction. China Satellite Navigation Conference (CSNC) 2017 Proceedings (Vol. II, pp. 271-282). Springer Singapore: Singapore.

Zhang, B., Jia, X., Sun, F., Xiao, K., \& Dai, H. (2019a). Performance of BeiDou-3 satellites: Signal quality analysis and precise orbit determination. Advances in Space Research, 64(3), 687-695. https://doi.org/10.1016/j.asr.2019.05.016.

Zhang, Z., Li, B., Nie, L., Wei, C., Jia, S., \& Jiang, S. (2019b). Initial assessment of BeiDou-3 global navigation satellite system: Signal quality, RTK and PPP. GPS Solutions, 23(4), 111. https://doi.org/10.1007/s10291-019-0905-4.

\section{Submit your manuscript to a SpringerOpen ${ }^{\odot}$ journal and benefit from:}

- Convenient online submission

- Rigorous peer review

- Open access: articles freely available online

- High visibility within the field

- Retaining the copyright to your article

Submit your next manuscript at $\boldsymbol{\nabla}$ springeropen.com 